\title{
Distal femural fractures. Surgical options
}

\author{
Pancrazio La Floresta, Salvatore Miceli, Giovanni Doronzo, \\ Massimiliano Borri \\ UOC Ortopedia e Traumatologia, Ospedale Antonio Cardarelli, Campobasso, Italy
}

\begin{abstract}
SUMMARY
Distal femur fractures are complex and relatively rare. Conservative treatment has now been abandoned. The purpose of surgical treatment is to obtain an anatomical reduction of the articular surface, restoring the coronal and sagittal axis of the skeletal segment with a stable osteosynthesis, thus avoiding post-operative immobilization and allowing for early physical therapy. The literature reports similar results with different kinds of osteosynthesis: anterograde or retrograde nailing, dynamic compression screw plate, locking compression plates, and blade plates. Not only the type of fracture, but also the experience of the single surgeon should be taken into account when choosing the type of osteosynthesis.
\end{abstract}

Key words: distal femur fracture, supracondylar and intercondylar fracture, internal fixation, external fixation

Received: December 20, 2019

Accepted: February 5, 2020

Correspondence

Massimiliano Borri

UOC Ortopedia e Traumatologia,

Ospedale Antonio Cardarelli, Contrada Tappino,

86100 Campobasso (CB), Italy

E-mail: massi.borri@hotmail.it

Conflict of interest

The Authors declare no conflict of interest

How to cite this article: La Floresta P, Miceli S, Doronzo G, et al. Distal femural fractures. Surgical options. Lo Scalpello 2020;34:83-8. https:// doi.org/10.36149/0390-5276-014

(C) Ortopedici Traumatologi Ospedalieri d'Italia (O.T.O.D.i.) 2020

\section{(c) (i) () $\Theta$}

This is an open access article distributed in accordance with the CC-BY-NC-ND (Creative Commons Attribution-NonCommercial-NoDerivatives 4.0 International) license. The article can be used by giving appropriate credit and mentioning the license, but only for non-commercial purposes and only in the original version. For further information: https://creativecommons.org/licenses/by-nc-nd/4.0/deed.en

\section{Introduction}

Fractures of the distal femur occur in the $9 \mathrm{~cm}$ proximal to the femoral tibial joint, they are infrequent and severe. The estimated frequency is $0.4 \%$ of all fractures and $3 \%$ of femoral fractures ${ }^{1}$. The occurrence is distributed following a bimodal model with a peak frequency in young males, around 30 years old, due to highenergy trauma, and in elderly women around the age of 70 by domestic accident ( $1 \mathrm{man} / 2$ women). In elderly subjects, these fractures are burdened by a morbidity and mortality similar to those of the proximal femur fractures. The rate of mortality increases when the fracture occurs on a previous knee prosthesis intervention or if the surgery is delayed beyond 96 hours $^{2}$. The non-operative treatment routinely used up until the 1970s resulted in failure in about $70 \%$ of cases ${ }^{3}$. Only with surgical intervention is it possible to obtain a stability such as to withstand the static loading forces on the bone and the dynamic muscular forces. For this reason, the non-surgical option is rare and can be considered only for poorly autonomous or bed bound patients, and in minimal or non-displaced fractures ${ }^{4}$.

Despite correct surgical treatment, the possible complications are frequent: aseptic pseudoarthrosis in $14 \%$ of cases; infection and septic pesudoarthrosis in $13 \%$ reaching $29 \%$ in exposed fractures; joint stiffness in $35 \%$ of cases, post-traumatic arthrosis in $50 \%$ of cases ${ }^{5}$.

Besides clinical examination and standard radiological examination, CT is recommended because $55 \%$ of these fractures are intra-articular ${ }^{1}$.

According to the $\mathrm{AO}$ classification, the distal femur fractures are 33A Extra articular: 1) simple; 2) wedge; 3) multifragmentary. 33B Partial articular: 1) lateral sagittal; 2) medial sagittal; 3 ) frontal (Hoffa fracture). $33 \mathrm{C}$ complete articular: 1) simple; 2) comminuted metaphyseal; 3) multifragmentary. 


\section{Discussion}

\section{Surgical options}

Surgical treatment aims to obtain anatomical reduction of the articular surface, restoring the sagittal and coronal axes of the skeletal segment with a stable osteosynthesis in order to avoid post-operative immobilization and allow for early physiotherapy. Surgical treatment of distal femur fractures led to a $32 \%$ reduction in unsatisfactory results compared to nonsurgical treatment ${ }^{6}$. In closed fractures, surgical approaches use internal fixation with anterograde intramedullary nailing or retrograde nailing, fixation with conventional plates or locking plates, blade plates and screws. The various techniques of internal fixation in Zlowodsky's work obtain similar results in terms of infections, pseudoarthrosis, fixation failure or revision. However, locking plates reduce infection, the procedure leads to an increase in revisions compared to conventional plates ${ }^{6}$. Intramedullary nailing prevents the extensive surgical exposure often required when using plates, also reducing bone devascularization that can cause infections and pseudoarthrosis. By contrast, to obtain a good reduction in comminuted fractures with severe joint involvement, vast exposure with an open reduction and plating become necessary. In exposed Gustilo type II and III fractures, urgent treatment with axial or circumferential external fixation with a subsequent conversion to internal osteosynthesis - if and when possible - is strongly indicated ${ }^{7}$.

\section{External fixation}

External fixation is indicated in exposed Gustilo type II and III fractures, or in polytraumatized patients by means of temporary stabilisation. Temporary external fixation allows bone lesion stabilization when serious soft tissue lesions have occurred, avoiding the potential risks related to internal osteosynthesis, and allowing adequate urgent treatment of the polytraumatized patient ${ }^{8}$. External fixation provides medical management and a Damage Orthopedic Control approach which reduces pain and facilitates treatment.

A satisfactory reduction is extremely difficult to achieve as the small lever arm in external fixation devices makes it difficult to control joint fragment. The use of bridging external fixation improves stability, but significantly increases the risk of stiffness if conversion to internal osteosynthesis is not carried out quickly ${ }^{9}$ (Fig. 1). On the other hand, good functional results have been reported with the Ilizarov technique associated with minimal intra-articular open synthesis in exposed type III fractures treated with external fixation until healing ${ }^{10}$.

In osteosynthesis with external fixation, it may be appropriate to implant the pins in the anterior femur to avoid subsequent contamination of the prospective plating site and to insert the pins at a sufficient distance from the fracture and the joint so as to limit the risk of infection ${ }^{9}$.

\section{Mono condylar fractures}

Exposed mono condylar fractures occur at a very low rate, and can be associated with patella fractures $(10 \%)$, anterior cruciate ligament injuries (10\%), contralateral tibial plate fractures ${ }^{11}$. Osteosynthesis is usually performed with screws placed perpendicular to the bone fracture wedge. Percutaneous osteosynthesis can be performed with minimal displacement. In Hoffa fractures, the knee joint is flexed during surgery to distally push the fragment of the condyle displaced above.

In a study by Maheshwari ${ }^{12}$, an analysis of radiographic and functional results in Hoffa fractures treated with Herbert headless screws compared with cannulated screws, a lower rate of complications was shown when using headless screws.

The synthesis with two $6.5 \mathrm{~mm}$ diameter screws would allow for a more rigid and stable fixation than the 2 or $4,4.5 \mathrm{~mm}$ cannulated screws in the synthetic bone model presented in the work of Kalafi ${ }^{13}$.

\section{Antegrade nailing}

In 33A extra articular fractures, antegrade intramedullary nailing allows closed osteosynthesis and not impacting the joint. The surgical procedure takes place at a distance from the of fracture allowing integral conservation of heamatoma; reduces risk of infection and prevents devascularization. This method allows the simultaneous treatment of bi- or multi-focal fractures with a relatively agile procedure obtaining optimal proximal stability. By contrast, distal fixation can be difficult, as it is the correction of the posterior inclination of the distal fragment. To obtain sufficient stability, the fragment's length should be a at least equal to the width of the condyle ${ }^{11,14}$. In Antegrade Nailing biomechanical studies have shown that to be able to withstand 1 million cycles of loading, it is necessary that the most proximal of the distal locking screws is located at least $3 \mathrm{~cm}$ distal to the fracture site ${ }^{15}$. The diameter of the nail is equally important for the stability of the distal fragment, in fact, distal cortical contact of the nail reduces the forces passed on to the locking system ${ }^{16}$.

\section{Retrograde intramedullary nailing}

Indications for retrograde nailing are 33A or 33C supracondylar fractures with little displacement. The procedure can be useful in ipsilateral hip arthroplasty or in supracondylar fracture in patients with certain types of knee prosthesis ${ }^{17}$. The nail is inserted intra-articularly to the front of the intercondylar notch in a minimally invasive way. In supracondylar fracture with a distal wedge long enough, the nail allows for a stable fixation with minimal surgical trauma. In diacondylar fractures a closed procedure is difficult to perform. The nail insertion point corresponds to the intercondylar fracture and there is an actual risk of increasing fragmentation. In these cases, before nail insertion, it is advisable to carry out a synthesis of the condyles with one or two cannulated screws introduced anteriorly to the 


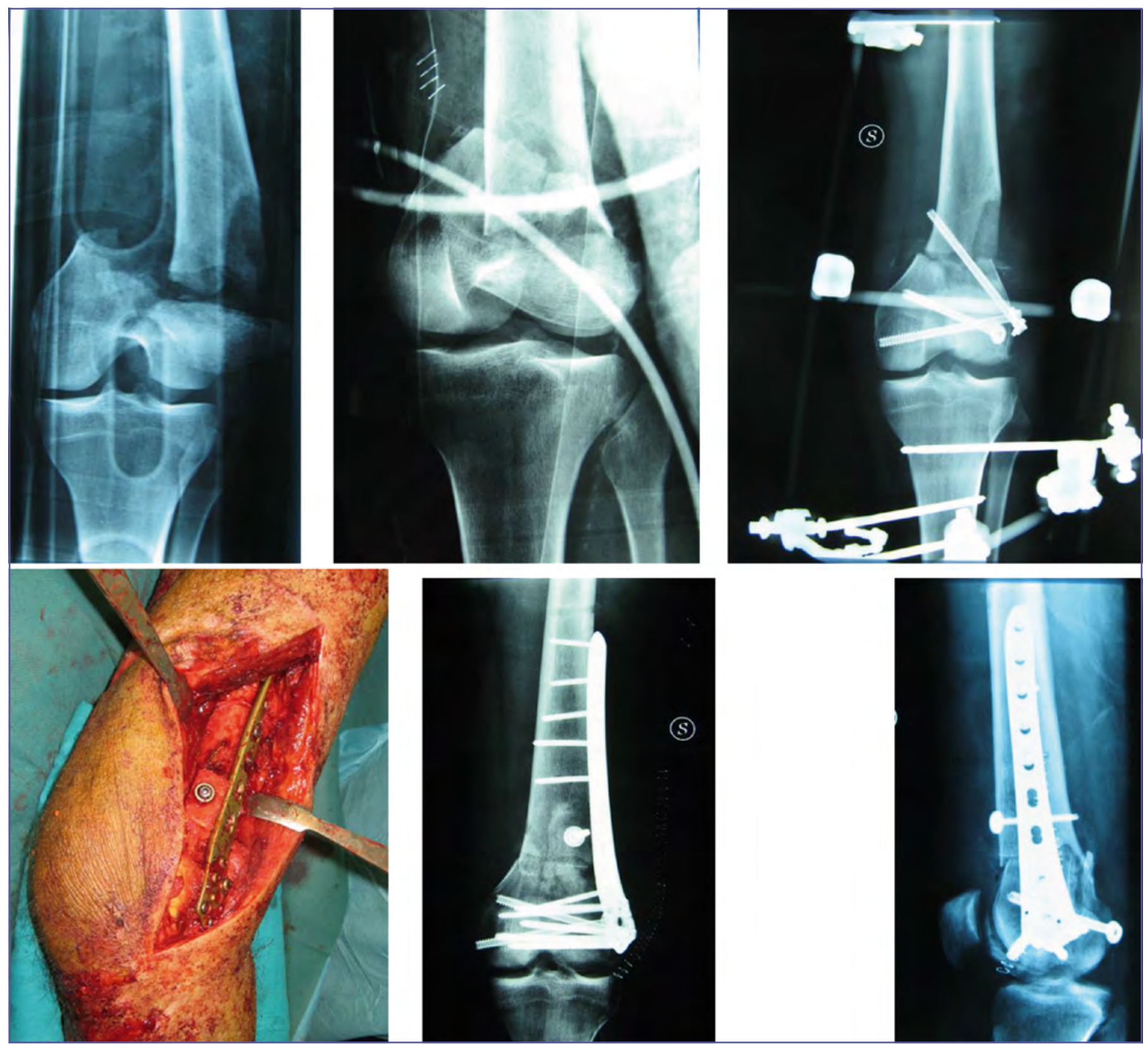

Figure 1. I.G. Male, 43-years-old, fracture $33 \mathrm{C} 1$ Gustilo III A. Temporary synthesis with external bridging fixator. Definitive synthesis with locking compression plate and bone graft.

nail point of entry to transform a type $\mathrm{C}$ fracture into a type A fracture ${ }^{18}$. Moreover, in complex supracondylar fractures, it is necessary to obtain good reduction before introducing the guide wire, in order to avoid relying on the nail for posterior and sagittal alignment of the fragments. The use of one or two Poller screws can guarantee a correct reduction by correctly directing the guide wire and the nail ${ }^{19}$. Importantly, the nail should be long enough to reach and exceed the isthmus of the femur to ensure good stability and a good distribution of forces.
The proximal locking should always use two screws to achieve sufficient mechanical stability. With the correct indication, retrograde nailing can lead to excellent results as described by Dileep ${ }^{20}$ who reported $81 \%$ excellent to good results in the treatment of 21 fractures (15 A1, 3 A2, 3 A3). Shiao Hao's ${ }^{21}$ biomechanical study demonstrates the importance of distal screws in improving stiffness of bone-nail construct. The absence of one of the two screws in the distal holes increases the stress on the free holes by $70 \%$. The distal-screws have a 
greater impact on the stability of the construct than the proximal ones. The stability of the nail bone construct increases by $40 \%$ in oblique fractures when using perifracture screws.

A comparative biomechanical analysis of retrograde intramedullary nails and plate systems showed lower torsional and axial stiffness of nails, but similar resistance to varus/ valgus stress ${ }^{22,23}$. Confuting the assumption of the retrograde intramedullary nail inadequacy in osteosynthesis of type $\mathrm{C}$ fractures, 33 months after the trauma. Seifer re-examined 44 patients treated with retrograde nail for type $\mathrm{A}$ and $\mathrm{C}$ fractures and found no significant differences in the results with a percentage of good to excellent results of $84 \%{ }^{24}$.

\section{Plate osteosynthesis}

Several plate types are available: blade plate, dynamic compression screw plate, traditional plates or locking compression plate.

The blade plate is an extremely stable osteosynthesis system, its use is indicated in extra articular, sagittal, supra- or intercondylar fractures, and allows to obtain good stability with medial fracture site compression. This method is limited by the difficulty in achieving correct positioning, poor resistance in osteoporotic bone and the systematic need to associate screws to obtain sufficient reduction and stabilization in diacondylar fractures. The blade is angled at $95^{\circ}$ to the plate and must be inserted parallel to the condyles axis to obtain a compression effect on the medial cortex once screwed onto the diaphyseal cortex. It must be positioned anterior to the Blumensatt line to avoid injuries to the cruciate ligaments, and it must not interfere with the femoral trochlea. The blade should not extend over the medial cortex of the condyle to avoid damaging the medial collateral ligament. The invasiveness of the distal blade prevents it from being positioned too distally; the distance between the joint surface and the blade should be about $15-20 \mathrm{~mm}^{25}$. This system, although valid, is no longer very popular as it is more complex than the most recent synthesis systems.

The dynamic compression screw plate (DCS) has similar indications to the blade plate. The angle between the plate and the cannulated distal screw is $95^{\circ}$, which allows a certain ease in positioning the screw. The screw conformation allows for interfragmentary compression in diacondylar fractures. However, the large screw hole causes a discrete loss of bone tissue; also in this case the distance between the cartilaginous surface of the femur and the screw must be at least $2 \mathrm{~cm}^{25}$.

Nowadays, anatomical locking compression plates are the most common osteosynthesis. The aim of this type of synthesis is to obtain excellent stability of the fracture by relying on the greater rigidity of the bone plate construct with locked screws compared to what can be obtained with a conventional plate. In these plates, the possibility of using both locked screws and conventional screws allows a good stability of the construct by exploiting both the possibility of compression of the distal section and the neutralization of forces. In supra- or trans- condylar fracture with low displacement, this minimally invasive technique reduces post-operative pain, ensures better preservation of soft tissues and reduces the risk of infection. However, for successful implantation it is essential to accurately follow the surgical technique to avoid errors that can lead to reduction deficits and mechanical problems. Especially when using a minimally invasive technique, special attention must be paid in reducing the fracture and correctly positioning the plate whose proximal end must not exceed the bone line. The distal positioning of the plate must be carried out with extreme care in order to avoid an excessively distal localization leading to a joint conflict. Likewise, distal periarticular screws must be directed with care and accuracy to avoid both penetration into the intercondylar notch, and subsequent conflict with the cruciate ligament - in case of posterior direction - and anterior protrusion in the femoral trochlea with damage to the patella. The number of screws and their relative position to the fracture site depends on the type of fracture; in unstable fractures, the screws are placed near the fracture to obtain greater stability, while in stable transverse fractures the screws can be placed further from the fracture to achieve greater elasticity of the system and ensure healing ${ }^{26}$. The rigidity of the bone plate construct is also significantly influenced by the distance between the plate and the bone with a notable reduction in resistance to compression and torsion, if the distance goes from 2 to $5 \mathrm{~mm}^{27}$.

In his work Ricci ${ }^{28}$ analyzed the risk factors for failure in the treatment of distal femoral fractures with locked plates showing that, while the type of fracture, open fracture, BMI, smoking, diabetes and patient age are factors independent of surgical choices, a statically significant reduction in failures is obtained when using relatively long plates with more than 8 holes.

Rodguez ${ }^{29}$ found a lower percentage of failures in constructs with medium stiffness compared to those that are excessively elastic or too rigid, as well as with titanium plates.

In his work Zlowodzki ${ }^{6}$ did not report significant differences in surgical outcomes of distal femoral fractures with blade plates, DCS, conventional or locking compression plates in terms of rate of pseudoarthrosis, infections, technical errors or revision. However, in the analysis of subgroups, compared to traditional implants, it is evident that the use of mini-invasive systems is associated with a reduction in the rate of infection and an increase in the number of revisions due to incorrect fixation. In his work, Hearther ${ }^{30}$ compared the results obtained in 33 cases of distal femoral fractures treated with a $95^{\circ}$ angled blade plate with 39 cases treated with the LCP system, while achieving good results with both systems, minor complications were reported with the $95^{\circ}$ blade plate.

In the publications of the "Canadian Orthopedic Trauma Society" ${ }^{31}$ and Kao ${ }^{32}$ the results obtained in distal femoral fracture treatment have been compared to those obtained with screws, DCS and LISS plates, and no advantages were found in the use of locking compression plates. 
To reduce the risk of mechanical failure with varus collapse, some authors, including Zhibiao ${ }^{33}$, Ziran ${ }^{34}$ and Fontenot ${ }^{35}$ suggested adding a medial plate in the osteosynthesis of superdiacondylar fractures in case of metaphyseal comminution. In his study, Zhibiao used a double plate in 12 of 60 cases having the indication for a double stabilization when, after having fixated the fracture with a lateral plate, the varus test of the construct is positive. With this method and a bone graft, no failure was reported in $91.7 \%$ of patients treated with a double plate.

\section{Knee arthroplasty}

In elderly patients with complex fractures, knee arthroplasties can be included as a therapeutic option. This procedure can be extremely complex, might require hinged prosthesis, and has a high mortality rate ${ }^{36}$.

\section{Conclusions}

The choice of the osteosynthesis method must be guided not only by the type of fracture, but also by the experience of the surgeon. The purpose of surgical treatment is to obtain an anatomical reduction of the articular surface, restoring the coronal and sagittal axis of the skeletal wedge with a stable osteosynthesis, thus avoiding post-operative immobilization and allowing for early physical therapy. If this result can be obtained with minimally invasive techniques that preserve the soft tissues as much as possible, these techniques should be preferred since they reduce the incidence of infections and facilitate the healing process. On the other hand, mini-invasiveness is a means and not an end, and therefore the use of DCS-type plate screws or $95^{\circ}$ blade plates, as well as double medial and lateral plates and possibly bone grafts, produces excellent results when treating complex and severely unstable comminuted fractures.

\section{References}

1 Court-Brown M, Caesar B. Epidemiology of adult fracture: a review. Injury 2006;37:691-7.

2 Philipp N, Streuber MD, William M, et al. Mortality after distal femur fractures in elderly patients. Clin Orthop Relat Res 2011;469:1188-96.

3 Nasr AM, Mc Leod I, Sabboubeh A, et al. Conservative or surgical management of distal femoral fractures. A retrospective study with a minimal five year follow-up. Acta Orthop Belg 2000;66:428-37.

4 Ehlinger M, Ducrot, Adam P, et al. Distal femur fractures. Surgical techniques and a review of the literature. Orthop Traumatol Surg Res 2013;99:353-60.

5 Asencio G. Les fractures del l'extrémité inferiéure du femur. Table ronde de la Sofcot. Rev Chir Orthop 1988;75(Suppl 1):168-83.

6 Zlowodzki M, Bhandari M, Marek DJ, et al. Operative treatment of acute distal femur fractures: systematic review of 2 comparative studies and 45 case series (1989 to 2005). J Orthop Trauma 2006;20:366-71.
7 Bonnevialle $\mathrm{P}$, Mansat $\mathrm{P}$, Cariven $\mathrm{P}$, et al. Fixation externe monoplan latéral des fractures fraîches du fémur: analyse critique de 53 cas. Rev Chir Orthop 2005;91:446-56.

8 Parekh AA, Smith WR, Silva S, et al. Treatment of distal femur and proximal tibia fractures with external fixation followed by planned conversion to internal fixation. J Trauma 2008;64:736-9.

9 Oh JK, Hwang JH, Sahu D, et al. Complications rate and pitfalls of temporary bridging external fixator in periarticular comminuted fractures. Clin Orthop Surg 2011;3:62-8.

10 Rakesc K, Soumya SM, Narendra J, et al. Primary Ilizarov external fixation in open grade III type $\mathrm{C}$ open distal femur fractures: our experience. J Clin Orthop Trauma 2019;10:928-33.

11 Chiron P. Fractures de l'extrémité Inférieure du femur de l'adulte. EMC. Appareil Locomoteur 2009:1-14.

12 Maheshwari V, Sharma SL, Divyanshu Goyal, et al. Clinical experience with management of Hoffa fractures using headless compression screw and headed screw. J Clin Orthop Trauma 2019;10:934-40.

13 Kalafi A, Hzelwood S, Curtiss S, et al. Fixation of the femoral condyles: a mechanical comparison of small an large fragment screw fixation. J Trauma 2008;64:740-4.

14 Kulkarni SG, Varshneya A, Kulkarni GS, et al. Anterograde interloking nailing for distal femoral fractures. J Orthop Surg (Hong Kong) 2012;20:48-54.

15 Antekeier SB, Burden RL Ir, Voor MJ, et al. Mechanical study of the safe distance between distal femoral fracture site and distal locking screws in anterograde intramedullary nailing. J Orthop Trauma 2005; 19:693-7.

16 Huang SC, Lin CC, Lin J. Increasing nail-cortical contact to increase fixation stability and decreased implantstarin in antegrade locked nailing of distal femoral fractures: a biomechanical study. J Trauma 2009;66:436-42.

17 Nizegorodcew T, Maccauro G, Pezzillo F, et al. Inchiodamento retrogrado di femore e di articolazione del ginocchio: analisi dei risultati clinici e revisione della letteratura. Giornale Italiano di Ortopedia e Traumatologia 2006;32(Suppl 1):5105-9.

18 Impagliazzo A, Lipsi A, Magistro L. Inchiodamento endomidollare retrogrado del femore. Lo Scalpello 2009;22:158-64.

19 Ostrum RF, Maurer JP. Distal third femur fractures treated with retrograde femoral nailing and blocking screws. J Orthop Trauma 2009;23:681-4.

20 Dileep KS, Mahesha K. Retrograde intramedullary Nailing for fractures of distal femur: a prospective study. Int J Res Orthop 2016;2:276-9.

21 Chen SH, Yu TC, Chang CH, et al. Biomechanical analysis of retrograde intramedullary nail fixation in distal fremoral fratures. Knee 2008;15:384-9.

22 David SM, Harrow ME, Peindl RD, et al. Comparative biomechanical analysis of supracondylar femur fracture fixation: locked intramedullary nail versus 95 -degree angled plate. J Orthop Trauma 1997;11:344-50.

23 Zlowodzki M, Williamson S, Cole PA, et al. Biomechanical evaluation of the less invasive stabilization system, angled blade plate, and retrograde intramedullary nail for the internal fixation of distal femur fractures. J Orthop Trauma 2004;18:494-502.

24 Seifert J, Stengel D, Matthes G, et al. Retrograde fixation of distal femoral fractures: results using a new nail system. J Orthop Trauma 2003;17:488-95. 
25 Dimitrov N. Tratment of fractures of the distal femoral overwies of ostheosynthesis fund. Trachia Journal of Sciences 2016;4:33343.

26 Stoffel K, Dieter U, Stachowiak G, et al. How can stability in locked internal fixators be controlled? Injury 2003;34:11-9.

27 Ahmad M, Nanda R, Bajwa AS, et al. Biomechanical testing of the locking compression plate: when does the distance between bon and implant significantly reduce construct stability? Injury 2007;38:358-64.

28 Ricci WM, Streubel PN, Morshed S, et al. Risk factors for failure of loked plate fixation of distal femur fractures: an analysis of 335 cases. J Orthop Trauma 2014;28:83-9.

29 Rodriguez EK, Boulton C, Weaver MJ, et al. Predictive factors of distal femoral fracture nonunion after lateral lockedplating: a retrospective multicenter case-control study of 283 fractures. Injury 2014;45:554-9. https://doi.org/10.1016/j.injury.2013.10.042

30 Heather A, Wes I. Comparison of the 95-degree angled blade plate and the locking condylar plate for the treatment of distal femoral fractures. J Orthop Trauma 2012;26:327-32.

31 Canadian Orthopaedic Trauma Society. Are locking costructs in distal femoral fractures always best? A prospective multicenter randomized controlled trial comparing the less invasive stabilization system with the minimally invasive dynamic condylar screw system. J Orthop Trauma 2016;30:e1-6.

32 Kao FC, Tu YK, Su JY, et al. Treatment of distal femoralfracture by minimally invasive percutaneous plate osteosynthesis: comparisonbetween the dynamic condylar screw and the less invasive stabilization system. J Trauma 2009;67:719-26.

33 Zhibiao B, Shichang G, Zhenming H, et al. Comparison of clinical efficacy of lateral and lateral and medial double - plating fixation of distal femoral fractures. Sci Rep 2018;8:4863.

34 Ziran BH, Rohde RH, Warton AR. Lateral and anterior plating of intra articular distal femoral fractures treated via an anterior approach. Int Orthop (SICOT) 2002;26:370-3.

35 Fontenot PB, Diaz M, Stops K, et al. Supplemetation of lateral Locked Plating for distal femur fracture: a biomechanical study. J Orthop Trauma 2019;33:642-8.

36 Appleton P, Moran M, Houshian S, et al. Distal femoral fractures treated by hinged total knee replacement in elderly patients. $\mathrm{J}$ Bone Joint Surg Br 2006;88:1065-70. 